\title{
PhySortR: a fast, flexible tool for sorting phylogenetic trees in R
}

Timothy G Stephens, Debashish Bhattacharya, Mark A Ragan, Cheong Xin Chan

A frequent bottleneck in interpreting phylogenomic output is the need to screen often thousands of trees for features of interest, particularly robust clades of specific taxa, as evidence of monophyletic relationship and/or reticulated evolution. Here we present PhySortR, a fast, flexible R package for classifying phylogenetic trees. Unlike existing utilities, PhySortR allows for identification of both exclusive and non-exclusive clades uniting the target taxa based on tip labels (i.e. leaves) on a tree, with customisable options to assess clades within the context of the whole tree. Using simulated and empirical datasets, we demonstrate the potential and scalability of PhySortR in analysis of thousands of phylogenetic trees without a priori assumption of tree-rooting, and in yielding readily interpretable trees that unambiguously satisfy the query. PhySortR is a command-line tool that is freely available and easily automatable. 


\section{PhySortR: a fast, flexible tool for sorting phylogenetic trees}

\section{2 in $\mathbf{R}$}

3 Timothy G. Stephens ${ }^{1}$, Debashish Bhattacharya ${ }^{2}$, Mark A. Ragan ${ }^{1}$ and Cheong Xin Chan ${ }^{1}$

$4 \quad{ }^{1}$ ARC Centre of Excellence in Bioinformatics, and Institute for Molecular Bioscience, The

5 University of Queensland, Brisbane, QLD 4072, Australia

$6{ }^{2}$ Department of Ecology, Evolution and Natural Resources, Rutgers University, New Brunswick, 7 NJ 08901, U.S.A.

8 Corresponding author:

9 Cheong Xin Chan

10 Institute for Molecular Bioscience, The University of Queensland, Brisbane, QLD 4072,

11 Australia

12 Email address: c.chan1@uq.edu.au 


\section{Abstract}

14 A frequent bottleneck in interpreting phylogenomic output is the need to screen often thousands

15 of trees for features of interest, particularly robust clades of specific taxa, as evidence of

16 monophyletic relationship and/or reticulated evolution. Here we present PhySortR, a fast,

17 flexible R package for classifying phylogenetic trees. Unlike existing utilities, PhySortR allows

18 for identification of both exclusive and non-exclusive clades uniting the target taxa based on tip

19 labels (i.e. leaves) on a tree, with customisable options to assess clades within the context of the

20 whole tree. Using simulated and empirical datasets, we demonstrate the potential and scalability

21 of PhySortR in analysis of thousands of phylogenetic trees without a priori assumption of tree-

22 rooting, and in yielding readily interpretable trees that unambiguously satisfy the query.

23 PhySortR is a command-line tool that is freely available and easily automatable.

\section{Introduction}

25 Phylogenomics increasingly involves the screening of thousands of phylogenetic trees using

26 specialised sorting algorithms that assign phylogenetic trees a classification based on features of 27 interest, e.g. strongly supported monophyletic relationships of taxa in question (i.e. the "target" taxa). Here, phylogenetic trees in flat files (e.g. Newick format) are sorted (i.e. classified) based on text-pattern matching. This principle is not to be confused with the tree sort process, common in computer science, of rearranging binary data elements in an ordered structure (Knuth 1971).

31 Currently available utilities, e.g. PhyloSort (Moustafa \& Bhattacharya 2008) and SICLE

32 (DeBlasio \& Wisecaver 2013) screen a set of phylogenetic trees for the presence of clades that

33 unite a set of user-defined target taxa (as indicated in tip labels, i.e. leaves, on the tree) based on

34 clade support that exceeds a defined threshold, and sort these trees accordingly; SICLE

35 (DeBlasio \& Wisecaver 2013) specifically identifies all nearest neighbours (sister clades) of a 
36 single user-defined target. However, these tools do not consider the proportion of non-target

37 leaves and overall taxon composition in a tree during the sorting process. Moreover, tools

38 implemented in a graphical user interface e.g. PhyloSort (Moustafa \& Bhattacharya 2008) do not

39 allow for automation of multiple analyses, thus limiting scalability.

$40 \quad$ Here we present PhySortR, a fast, flexible R package for screening and sorting

41 phylogenetic trees. The command-line package provides the quick and highly flexible sortTrees

42 function, allowing for screening (within a tree) for "Exclusive" clades that contain only the target

43 leaves and/or "Non-Exclusive" clades that include a defined portion of non-target leaves. Using

44 simulated data, we assess the runtime of PhySortR based on the number of trees and the number

45 of leaves within a tree, and demonstrate the potential of PhySortR in the analysis of multiple,

46 large-scale empirical datasets.

\section{Materials \& Methods}

\section{Rationale and basic principles of PhySortR}

Figure 1 shows four examples of tree topologies and their corresponding features relevant to the sorting process of PhySortR, each with a target clade identified as Clade Z; the first tree (Figure 1A) is an empirical tree of a putative sodium/sulphate symporter protein from an earlier study (Bhattacharya et al. 2013), on which the other three hypothetical topologies (Figures 1B-1D) are based. PhySortR allows the user to specify one or more target terms using the target.groups argument, providing that the leaves (tree-tip labels in the Newick files) are named consistently across all input trees; this is a simple string-matching exercise, i.e. the terms specified here determine taxon-level resolution of targets. In the examples shown in Figure 1, target.groups $=$ "Rhodophyta,Viridiplantae,Stramenopiles". The minimum support for a clade (min.support) can 
58 refer to bootstrap, Bayesian posterior probability, or any other measure of support. Here, no prior

59 assumption of tree root is made (i.e. all trees are treated as unrooted), thus clade membership on

60 both sides of each node is considered. For instance, the node support for Clade $Z$ (bootstrap 99\%)

61 in Figure 1A is also considered as the support for the opposing bacterial clade (Proteobacteria +

62 Cyanobacteria) on the tree. This is distinct from the assumption of the lowest common ancestor

63 for a clade in PhyloSort (Moustafa \& Bhattacharya 2008), in which subtree-rooting could be

64 invoked during a search.

65 Existing utilities identify clades of interest without considering the occurrence of the target

66 leaves elsewhere on the tree. For example, when assessing a target clade in a 30-leaf tree,

67 PhyloSort will positively identify both (a) a robust 24-member clade (Clade $Z$ in Figure 1A) and

68 (b) a robust four-member clade (Clade $Z$ in Figure 1B), although (a) is the more-convincing

69 evidence of a close association between the targets and of greater biological significance; here

70 most of the target group is contained within Clade $Z$ (Figure 1A), compared to the scenario in

71 Figure 1B, in which most of the target group, with no clear evidence of overall monophyly, is

72 placed externally to robust Clade $Z$. To address this issue, PhySortR allows the user to define

73 min.prop.target, the minimum required proportion of target(s) present in a clade relative to the

74 total number of target(s) found in a tree. The default value of min.prop.target is set at 0.7 as

75 guidance. At 1.0, a strict monophyletic relationship of the target group is enforced (i.e. no target

76 leaves occurring elsewhere on a tree). When the value is set too low (e.g. at 0.1 ), one would

77 identify a target clade that is more-narrowly defined (e.g. Clade $Z$ in Figure 1B); such a clade

78 could have limited biological significance. This option provides users the flexibility to design a

79 query that can be tailored to address specific biological questions, e.g. to identify a clade of

80 interest defined as narrowly or as broadly as desired. 
Alternatively, the user may wish to screen for a robust clade that contains the target groups and a small proportion of "interrupting" non-target leaves, e.g. a 24-member clade consisting of 23 leaves from the targets Rhodophyta, Viridiplantae, and Stramenopiles, as well as one from a non-target leaf, Haptophyceae (Clade $Z$ in Figure 1C). Whereas the haptophyte is "interrupting” the otherwise exclusive clade of Rhodophyta + Viridiplantae + Stramenopiles, the association among the targets is still of interest and the presence of the haptophyte might be readily explained by lateral gene transfer (LGT) due to plastid endosymbiosis (e.g. Bowler et al. 2008; Chan et al. 2011b; Howe et al. 2008). Composite clades such as these are considered "NonExclusive" (Chan et al. 2011b) and are not identified by existing sorting tools. The concept of exclusivity (Figure 1A) versus non-exclusivity (Figure 1C) of clades in tree sorting has proven crucial in a number of genome-wide studies that have investigated the impact of LGT on the evolution of diverse algae and protists (e.g. Bhattacharya et al. 2013; Chan et al. 2011b; Curtis et al. 2012; Price et al. 2012). In addition to biological implications (e.g. LGT or genetic recombination), by allowing the presence of non-targets in a clade, the non-exclusive clades are also useful in identifying the association of a target group against the backdrop of phylogenetic artefacts (Stiller 2011) that would weaken an otherwise strong phylogenetic signal, e.g. unbalanced taxon sampling (or missing taxa) (Rosenberg \& Kumar 2003; Sanderson et al. 2010), long-branch attraction (Felsenstein 1978), or contamination. PhySortR identifies both types of clade based on the proportion of target versus non-target leaves using the option clade.exclusivity. At the default setting (clade.exclusivity $=0.9$ ), the minimum proportion of

101 target leaves within a "Non-Exclusive" clade is 0.9 , thus the maximum proportion of non-target

102 leaves allowed in the clade is 0.1 (i.e. 1 minus 0.9 ). For instance, the proportion of target leaves 103 within Clade $Z(0.79)$ in Figure 1D does not satisfy the criterion of clade.exclusivity of 0.9; this 
104 clade is therefore not considered as "Non-Exclusive" at the default setting. This option accepts

105 any value $<1.0$, and is applicable only for sorting "Non-Exclusive" clades (see below); at 1.0 (no

106 non-target leaves allowed), the clade is considered "Exclusive".

\section{Sorting of phylogenetic trees}

108 In PhySortR, sortTrees is the function for sorting phylogenetic trees; the basic algorithm is

109 shown in Figure 2, and all available arguments are detailed in Table 1. To run sortTrees, the user

110 must aggregate all phylogenetic trees to be sorted into a single directory. All tree files must have

111 an identical file extension (see extension; Table 1) and can be in either standard or extended

112 Newick (Cardona et al. 2008) format.

113 The target.groups parameter (Table 1) is the only compulsory argument; all other

114 arguments have defaults that the function will use if an alternative is not provided. To avoid

115 ambiguity, the terms passed to the function are matched to a tree's tip labels by exact substring-

116 matching. Multiple terms passed to the function must be separated by a comma (e.g.

117 "Rhodophyta,Viridiplantae,Stramenopiles") and must be sufficiently specific in the dataset for

118 the purpose of the screening. For instance, "plantae" and "Viridiplantae" might not be

119 appropriate in a single query because all tips that are identified by "Viridiplantae" will also be

120 identified by "plantae".

121 Regardless of which parameters are passed to the mode argument, the function will always

122 return a list of the trees that have been identified as containing clades that meet the specified

123 criteria. If the move (mode $=$ " $m$ ") or copy (mode $=$ " $c$ ") command is given, subdirectories will

124 be created in out.dir that contain trees with a particular clade, i.e. the directory out.dir/Exclusive/

125 will be created for the trees with "Exclusive" clades and out.dir/Non_Exclusive/ for trees with 
126 "Non-Exclusive" clades. If the function is instructed to search for "Exclusive" trees it will also

127 return trees that contain only target leaves, termed "All Exclusive" trees. These trees are a subset

128 of "Exclusive" trees and will be transferred to a subdirectory (if the move/copy parameter is

129 given) within the "Exclusive" directory i.e. out.dir/Exclusive/All_Exclusive.

130 The clades.sorted parameter can be used to change the types of clades that the function

131 will search for. For example if clades.sorted = " $E$ " is given, the function will only search for

132 trees that have "Exclusive" clades, but if the default value of clades.sorted = "NE,E" is given, the

133 function will search for both "Exclusive" and "Non-Exclusive" clades. During each run the

134 function will create a $\log$ file, called "out.dir.log", in the in.dir directory. This file will contain

135 information about each identified clade, e.g. the names of the leaves in the clade, the support for

136 the clade, the proportion of "interrupting" leaves, and so forth.

\section{Conversion of extended Newick format}

138 Newick format is a standardised, machine-readable, plain-text representation of phylogenetic

139 trees (http://evolution.genetics.washington.edu/phylip/newick doc.html) that has been widely

140 adopted in phylogenetic software. This format was later modified to incorporate more-complex

141 network information such as hybrid nodes, in the form of extended Newick (eNewick) format;

142 see Cardona et al. (2008) for details. The two formats are however very similar, as shown in the

143 example tree topology in Figure 3. In Newick, the support value for a node precedes its branch

144 length, separated by a colon. In eNewick, the support value for a node, enclosed in square

145 brackets, is placed after its branch length (Figure 3). Most phylogenetic programs accept trees

146 only in the Newick format, but more-recent programs of phylogenetic inference generate the tree

147 output in eNewick format by default. Taking this into consideration, PhySortR provides the 
148 convert.eNewick function that takes a single phylogenetic tree in eNewick format and returns the

149 same tree in Newick format. This function in isolation can be used as a general-purpose tool for

150 converting phylogenetic trees in eNewick format into a format that is usable by the popular

151 phylogenetic packages in R, ape (Paradis et al. 2004) and phytools (Revell 2012).

\section{Simulation of phylogenetic trees}

153 To test the scalability of the PhySortR package we simulated benchmarking datasets composed

154 of a given number of trees $(N)$ and leaves per tree $(X)$; see Supplementary Figure S1 for detail.

155 All simulated trees are in the eNewick format. To simulate a tree with $X=100$, we used a base

156 phylogenetic tree with $1.05 X$ tips, i.e. 105 tips. An "Exclusive" 20-leaf target clade (i.e. $0.2 X$ ) is

157 defined, tip labels of other non-target leaves are swapped (at random), following which $0.05 X$

158 (i.e. 5) of the overall tree branches (external to the target clade) chosen at random were removed 159 using phytools, resulting in the final tree of size $X$. This tree was then replicated up to $N$ number 160 of trees as per our experimental design below.

161 Simulation of trees at different $X$ follows the same strategy as per above, and for negative

162 controls, the target clade was simply omitted. For the first analysis, we generated sets of input

163 trees at $N=1000,2000,4000,6000,8000$ and 10000 (each tree with $X=100$; Supplementary

164 Data S1). For the second analysis, we generated sets of input trees $(N=1000)$ at tree size $X=$

$165100,200,300,400$ and 500 (each tree of distinct size $X$ is available as Supplementary Data S2).

166 For the purpose of assessing scalability, all tree topologies are identical within a set of $N$ trees,

167 i.e. a single technical replicate (Supplementary Figure S1). All benchmark analyses were carried 168 out with 100 technical replicates (i.e. each replicate with a distinct base tree topology), on a 
169 desktop computer $\left(2.5 \mathrm{GHz}\right.$ Intel ${ }^{\circledR}$ Core $^{\mathrm{TM}}$ i5 with $4 \mathrm{~GB}$ memory). The $\mathrm{R}$ script for simulating

170 these topologies and the corresponding templates are available as Supplementary Data S3.

171 Comparative assessment of PhySortR versus PhyloSort

172 As input, here we used 897 empirical protein trees from an earlier study (Chan et al. 2011a).

173 Using PhySortR and PhyloSort (http://phylosort.sourceforge.net/), we screened for trees that

174 contain a strongly supported (bootstrap $\geq 90 \%$ ) exclusive clade of Viridiplantae + Stramenopiles.

175 The closest equivalent parameter settings were used for PhySortR (target.groups $=$

176 "Viridiplantae,Stramenopiles", min.support $=90$, min.prop.target $=1.00$, clades. sorted $=$ "E")

177 and PhyloSort $\left(\right.$ Taxa regexp $=\left(\left[{ }^{\wedge}\right]+{ }_{-}\left[{ }^{\wedge}\right]+\right){ }^{*}$, query taxa group $1=$ all Viridiplantae leaves,

178 query taxa group $2=$ all Stramenopiles leaves, Min bootstrap =90, Exclusive mode). For

179 PhyloSort, independent analysis was done with the option Root by outgroup (PhyloSort-root

180 hereinafter) and without (PhyloSort-no-root).

\section{Implementation and availability of PhySortR}

182 PhySortR depends on two other phylogenetic packages in R, ape (Paradis et al. 2004) and

183 phytools (Revell 2012). All three packages require R version 3.0 or above to function, and they

184 can be installed directly from CRAN in the R environment (see Supplementary Text S1 for

185 detail). PhySortR is freely available as a platform-independent R package from the

186 Comprehensive R Archive Network at https://cran.r-project.org/web/packages/PhySortR/.

187 Several examples are provided with the R package.

\section{Results \& Discussion}

189 Figure 4 shows the runtime of PhySortR relative to the number of trees $(N)$ to be sorted and the

190 number of leaves $(X)$ within a tree. As with any utility (DeBlasio \& Wisecaver 2013; Moustafa 
191 \& Bhattacharya 2008), the runtime of PhySortR is dependent on $N$ and $X$. We observed that the

192 runtime scales linearly with $N$ (Figure 4A) and superlinearly with $X$ (Figure 4B). In the extreme

193 case, sorting through 10,000 trees of $X=100$ took $<400$ seconds ( $~ 6.7$ minutes), and sorting

194 through 1,000 trees of $X=500$ took $<350$ seconds ( $\sim 5.8$ minutes). We observed negligible

195 differences in runtime with negative controls (trees containing no identifiable clades) as input,

196 compared to the test set in Figure 4B. Our findings demonstrate the potential of PhySortR in

197 analysis of multiple, large-scale datasets.

Unlike PhyloSort, no prior assumption of tree-rooting is made in PhySortR. To illustrate the impact of tree-rooting assumption on the sorting process, we performed sorting among 897 empirical protein trees (Chan et al. 2011a) using PhySortR, and compared them to PhyloSort with and without the rooting option (i.e. PhyloSort-root and PhyloSort-no-root; see Methods for detail). Here, in the search for an "Exclusive" clade of Viridiplantae + Stramenopiles with bootstrap $\geq 90 \%, 18,33$ and 46 trees were identified using PhyloSort-no-root, PhySortR and PhyloSort-root (Figure 5A). In Figure 5B, identification of the target clade (bootstrap 98\%) is straightforward in all methods; all methods also successfully recovered the same ten "All Exclusive" trees (containing only leaves of Viridiplantae + Stramenopiles). Figure 5C shows a

207 tree that is identified only using PhyloSort-root. Here the target clade is positively identified due to (the enabled) subtree-rooting of non-target leaves, not based on clade support. The absence of other closely related leaves in the tree could reinforce the association of Viridiplantae and

210 Stramenopiles (i.e. the tree might be biologically meaningful), but the bootstrap 61\% (Figure 5C)

211 is below the specified threshold (90\%) in the search; thus this tree is not recovered using

212 PhySortR and PhyloSort-no-root. Figure 5D shows a tree that is identified using PhySortR and

213 PhyloSort-root, but not PhyloSort-no-root. Here the condition of target clade support is satisfied 
214 (i.e. bootstrap 98\%; Figure 5D), but with the subtree-rooting option disabled, PhyloSort did not

215 recover this tree in the search; this appears to be a false negative. Whereas the results from

216 PhyloSort and PhySortR are not directly comparable, our results demonstrate the impact of the

217 tree-rooting assumption on the sorting results between the two programs. This underlying

218 assumption in PhyloSort could lead to under- or over-estimation of the number of positive

219 identifications; instances of false positives and false negatives will need to be manually verified

220 based on the research question. Here, PhySortR yields readily interpretable trees (e.g. Figure 5B

221 and 5D) that unambiguously satisfy the query requirements.

222

Furthermore, the algorithm of PhySortR (Figure 2) is distinct from existing utilities in two

key aspects: PhySortR considers in a tree (a) both exclusive and non-exclusive clades, and (b) a

224 clade of interest within the context of overall taxon composition. Both PhySortR and PhyloSort

225 were designed as tools for hypothesis testing, e.g. to identify a clade of target leaves as putative

226 evidence of genetic exchange and/or transfer. In comparison, SICLE was designed for a

227 fundamentally different task, the screening of all possible sister clades to a single target group

228 (instead of two or more targets as allowed in PhySortR and PhyloSort) as a tool for hypothesis

229 generation. These three programs are implemented in different programming languages, i.e.

230 PhySortR in R, PhyloSort in Java and SICLE in C++. The sorting process of phylogenetic trees

231 is therefore dependent not only on parameter settings but also implementation and hardware;

232 comparing computation time and results among these tools is not straightforward.

233 PhySortR is an R implementation based on the basic sorting principles of Chan et al.

234 (2011b) that has been widely adopted in other phylogenomic studies (Bhattacharya et al. 2013;

235 Curtis et al. 2012; Price et al. 2012). PhySortR incorporates existing functionalities and data 
236 structures in the commonly used phylogenetic packages ape (Paradis et al. 2004) and phytools

237 (Revell 2012), allowing for streamlined interoperability within the $\mathrm{R}$ environment. Whereas ape

238 and phytools accept only Newick as input, PhySortR accepts tree files in both Newick and

239 eNewick (Cardona et al. 2008) formats. The R platform (R Core Team 2015) is open source,

240 platform-independent, and broadly accessible to researchers, with continued support. In addition,

241 functional modularity and the command-line interface of PhySortR enable batch automation and

242 workflow integration.

\section{Acknowledgement}

244 We thank two anonymous reviewers for their constructive comments and suggestions.

\section{Supplementary Material}

246 Supplementary Data S1. Simulated trees with varying $N$ at fixed $X=100$.

247 Supplementary Data S2. Simulated trees with varying $X$ at fixed $N=1000$.

248 Supplementary Data S3. R script and templates for simulating tree topologies in this study.

249 Supplementary Text S1. Installation notes for PhySortR.

250 Supplementary Figure S1. Simulation strategy for generating phylogenetic tree sets.

251 References

252 Bhattacharya D, Price DC, Chan CX, Qiu H, Rose N, Ball S, Weber AP, Arias MC, 253 Henrissat B, Coutinho PM, Krishnan A, Zauner S, Morath S, Hilliou F, Egizi A, 
Perrineau MM, and Yoon HS. 2013. Genome of the red alga Porphyridium purpureum. Nature Communications 4:1941. 10.1038/ncomms2931.

256

Bowler C, Allen AE, Badger JH, Grimwood J, Jabbari K, Kuo A, Maheswari U, Martens C, Maumus F, Otillar RP, Rayko E, Salamov A, Vandepoele K, Beszteri B, Gruber A, Heijde M, Katinka M, Mock T, Valentin K, Verret F, Berges JA, Brownlee C, Cadoret JP, Chiovitti A, Choi CJ, Coesel S, De Martino A, Detter JC, Durkin C, Falciatore A, Fournet J, Haruta M, Huysman MJ, Jenkins BD, Jiroutova K, Jorgensen RE, Joubert Y, Kaplan A, Kröger N, Kroth PG, La Roche J, Lindquist E, Lommer M, Martin-Jézéquel V, Lopez PJ, Lucas S, Mangogna M, McGinnis K, Medlin LK, Montsant A, Oudot-Le Secq MP, Napoli C, Obornik M, Parker MS, Petit JL, Porcel BM, Poulsen N, Robison M, Rychlewski L, Rynearson TA, Schmutz J, Shapiro H, Siaut M, Stanley M, Sussman MR, Taylor AR, Vardi A, von Dassow P, Vyverman W, Willis A, Wyrwicz LS, Rokhsar DS, Weissenbach J, Armbrust EV, Green BR, Van de Peer Y, and Grigoriev IV. 2008. The Phaeodactylum genome reveals the evolutionary history of diatom genomes. Nature 456:239-244. 10.1038/nature07410.

Cardona G, Rossello F, and Valiente G. 2008. Extended Newick: it is time for a standard representation of phylogenetic networks. BMC Bioinformatics 9:532. 10.1186/1471-2105$9-532$.

Chan CX, Reyes-Prieto A, and Bhattacharya D. 2011a. Red and green algal origin of diatom membrane transporters: insights into environmental adaptation and cell evolution. PLoS ONE 6:e29138. 10.1371/journal.pone.0029138. 
275

276

277

278

279

280

281

282

283

284

285

286

287

288

289

290

291

292

293

294

295

296

Chan CX, Yang EC, Banerjee T, Yoon HS, Martone PT, Estevez JM, and Bhattacharya D. 2011b. Red and green algal monophyly and extensive gene sharing found in a rich repertoire of red algal genes. Current Biology 21:328-333. 10.1016/j.cub.2011.01.037.

Curtis BA, Tanifuji G, Burki F, Gruber A, Irimia M, Maruyama S, Arias MC, Ball SG, Gile GH, Hirakawa Y, Hopkins JF, Kuo A, Rensing SA, Schmutz J, Symeonidi A, Elias M, Eveleigh RJ, Herman EK, Klute MJ, Nakayama T, Oborník M, Reyes-Prieto A, Armbrust EV, Aves SJ, Beiko RG, Coutinho P, Dacks JB, Durnford DG, Fast NM, Green BR, Grisdale CJ, Hempel F, Henrissat B, Höppner MP, Ishida K, Kim E, Kořený L, Kroth PG, Liu Y, Malik SB, Maier UG, McRose D, Mock T, Neilson JA, Onodera NT, Poole AM, Pritham EJ, Richards TA, Rocap G, Roy SW, Sarai C, Schaack S, Shirato S, Slamovits CH, Spencer DF, Suzuki S, Worden AZ, Zauner S, Barry K, Bell C, Bharti AK, Crow JA, Grimwood J, Kramer R, Lindquist E, Lucas S, Salamov A, McFadden GI, Lane CE, Keeling PJ, Gray MW, Grigoriev IV, and Archibald JM. 2012. Algal genomes reveal evolutionary mosaicism and the fate of nucleomorphs. Nature 492:59-65. 10.1038/nature11681.

DeBlasio D, and Wisecaver J. 2013. SICLE: A high-throughput tool for extracting evolutionary relationships from phylogenetic trees. arXiV:1303.5785.

Felsenstein J. 1978. Cases in which parsimony or compatibility methods will be positively misleading. Systematic Zoology 27:401-410. 10.2307/2412923.

Howe CJ, Barbrook AC, Nisbet RE, Lockhart PJ, and Larkum AW. 2008. The origin of plastids. Philosophical Transactions of the Royal Society of London, Series B: Biological Sciences 363:2675-2685. 10.1098/rstb.2008.0050. 
297 Knuth DE. 1971. Optimum binary search trees. Acta Informatica 1:14-25. 10.1007/BF00264289.

299

300

301

302

303

Moustafa A, and Bhattacharya D. 2008. PhyloSort: a user-friendly phylogenetic sorting tool and its application to estimating the cyanobacterial contribution to the nuclear genome of Chlamydomonas. BMC Evolutionary Biology 8:6. 10.1186/1471-2148-8-6.

Paradis E, Claude J, and Strimmer K. 2004. APE: Analyses of Phylogenetics and Evolution in R language. Bioinformatics 20:289-290. 10.1093/bioinformatics/btg412.

Price DC, Chan CX, Yoon HS, Yang EC, Qiu H, Weber AP, Schwacke R, Gross J, Blouin NA, Lane C, Reyes-Prieto A, Durnford DG, Neilson JA, Lang BF, Burger G, Steiner JM, Löffelhardt W, Meuser JE, Posewitz MC, Ball S, Arias MC, Henrissat B, Coutinho PM, Rensing SA, Symeonidi A, Doddapaneni H, Green BR, Rajah VD, Boore J, and Bhattacharya D. 2012. Cyanophora paradoxa genome elucidates origin of photosynthesis in algae and plants. Science 335:843-847. 10.1126/science.1213561.

R Core Team. 2015. R: A Language and Environment for Statistical Computing.R Foundation for Statistical Computing, Vienna, Austria.

Revell LJ. 2012. phytools: an R package for phylogenetic comparative biology (and other things). Methods in Ecology and Evolution 3:217-223. 10.1111/j.2041-210X.2011.00169.x.

Rosenberg MS, and Kumar S. 2003. Taxon sampling, bioinformatics, and phylogenomics. Systematic Biology 52:119-124. 10.1080/10635150390132894. 
316 Sanderson MJ, McMahon MM, and Steel M. 2010. Phylogenomics with incomplete taxon coverage: the limits to inference. BMC Evolutionary Biology 10:155. 10.1186/1471-214810-155.

319

320

321

322

\section{3}

324

325

326

327

328

329

330

331

332

333

334

335

336

337

Stiller JW. 2011. Experimental design and statistical rigor in phylogenomics of horizontal and endosymbiotic gene transfer. BMC Evolutionary Biology 11:259. 10.1186/1471-2148-11259.

\section{Figure legends}

Figure 1: The effect of minimum target proportion and clade exclusivity on a tree. Tree topologies and their corresponding features relevant to min.prop.target and clade.exclusivity in PhySortR, with a target clade $Z$. The tree of a sodium/sulphate symporter protein (A) contains a strongly supported "Exclusive" clade $Z$ of Rhodophyta + Viridiplantae + Stramenopiles. Different scenarios are shown using hypothetical tree topologies, for (B) a low target proportion within target clade, (C) a "Non-Exclusive" target clade with a high extent of clade exclusivity, and (D) a low extent of clade exclusivity. A summary of the leaf composition corresponding to key selection criteria of PhySortR for each tree is shown at the bottom.

\section{Figure 2: Overview of the sorting algorithm in PhySortR.}

\section{Figure 3: A phylogenetic tree represented in standard and extended Newick formats.}

Figure 4: Benchmarking results of PhySortR using simulated data. The mean runtime of

$$
\text { PhySortR is shown for analysis across datasets (A) with different numbers of trees, } N \text {, and (B) }
$$
with different numbers of leaves per tree, $X$. Values of runtime (in second) are mean across 100 replicates, error bars indicate the standard deviation of the mean. 
338 Figure 5: Trees sorted using PhySortR and PhyloSort. The Venn diagram depicting number

339 of trees identified using PhySortR, PhyloSort-root and PhyloSort-no-root is shown in (A). An

340 example of tree output for trees that are identified in (B) all cases, (C) only PhyloSort-root, and

341 (D) all but PhyloSort-no-root are shown.

342 Table

343 Table 1: List of arguments within sortTrees function in PhySortR. 


\section{Figure 1 (on next page)}

The effect of minimum target proportion and clade exclusivity on a tree.

Tree topologies and their corresponding features relevant to min.prop.target and clade.exclusivity in PhySortR, with a target clade Z. The tree of a sodium/sulphate symporter protein $(A)$ contains a strongly supported "Exclusive" clade $Z$ of Rhodophyta + Viridiplantae + Stramenopiles. Different scenarios are shown using hypothetical tree topologies, for (B) a low target proportion within target clade, (C) a "Non-Exclusive" target clade with a high extent of clade exclusivity, and (D) a low extent of clade exclusivity. A summary of the taxon composition corresponding to key selection criteria of PhySortR for each tree is shown at the bottom. 
A Proteobacteria-marine g-proteobacterium HTCC2143 gi119477603

- Proteobacteria-Alkalilimnicola ehrlichii MLHE-1 gi114321494

19 43-Proteobacteria-Geobacter loveyi SZ gi189424589

Cyanobacteria-Synechococcus sp PCC7002 gi170077416

99 - Viridiplantae-Ostreococcus tauri jgi20378

99 - Viridiplantae-Ostreococcus RCC809 jgi39689

99 100_-Viridiplantae-Ostreococcus lucimarinus CCE9901 gi145352770

99- Viridiplantae-Ostreococcus lucimarinus jgi39202

Viridiplantae-Micromonas CCMP1545 jgi31122

68 - Viridiplantae-Micromonas RCC299 jgi79434

- - Viridiplantae-Micromonas RCC299 jgi99087

62 _ Viridiplantae-Micromonas RCC299 jgi66391

100 - Viridiplantae-Ostreococcus lucimarinus jgi1343

- Viridiplantae-Ostreococcus tauri jgi22319

68 82- Viridiplantae-Ostreococcus RCC809 jgi65597

_ Stramenopiles-Thalassiosira pseudonana CCMP1335 gi224002755

100 - Stramenopiles-Thalassiosira pseudonana CCMP1335 gi224013124

74 - Stramenopiles-Phaeodactylum tricomutum CCAP10551 gi219125414

92 - Stramenopiles-Phaeodactylum tricornutum CCAP 0551 gi219125416

100 - Viridiplantae-Chlamydomonas reinhardtii gi159478811

- Viridiplantae-Volvox carteri jgi59271

$95 \quad 39$ Rhodophyta-Porphyra haitanensis 1152884152

Lhodophyta-Cyanidioschyzon merolae CMM179C

99 Rhodophyta-Porphyridium purpureum Contig14254_2

100 Rhodophyta-Porphyridium purpureum Contig12409 3

$65-$ Rhodophyta-Porphyridium purpureum GCDJ7DB01EWRWK_1

${ }_{63}-$ Rhodophyta-Porphyridium purpureum Contig3350_1

\section{C} Proteobacteria-marine g-proteobacterium

\section{0 - Proteobacteria-Marinobacter $s p$}

33 - Proteobacteria-Marinobacter algicola

- Proteobacteria-Alkalilimnicola ehrlichi

19 43-Proteobacteria-Geobacter loveyi

- Cyanobacteria-Synechococcus $s p$

99 Viridiplantae-Ostreococcus tauri 1 1

- Viridiplantae-Ostreococcus RCC809 1

99 100_ Viridiplantae-Ostreococcus lucimariñus_1

99L Viridiplantae-Ostreococcus Iucimarinus_2 - Viridiplantae-Micromonas CCMP1545

- Viridiplantae-Micromonas RCC299 1

68 91-Viridiplantae-Micromonas RCC299_2

100100 91 — Viridiplantae-Micromonas RCC299 3

62 Viridiplantae-Micromonas RCC299_4

100_Viridiplantae-Ostreococcus lucimarinus_3

- Viridiplantae-Ostreococcus tauri 2

68 82L Viridiplantae-Ostreococcus RCC809_2 Stramenopiles-Thalassiosira pseudonana 1

100 Stramenopiles-Thalassiosira pseudonana_2

74 -Stramenopiles-Phaeodactylum tricornutum_ 1

92 - Haptophyceae-Emiliania huxleyi

100 -Viridiplantae-Chlamydomonas reinhardtii

$95 \quad$ Viridiplantae-Volvox carteri

Rhodophyta-Cyanidioschyzon merolae

99 Rhodophyta-Porphyridium purpureum_1

100 Rhodophyta-Porphyridium purpureum_2

65 - Rhodophyta-Porphyridium purpureum_3

63 Rhodophyta-Porphyridium purpureum_4
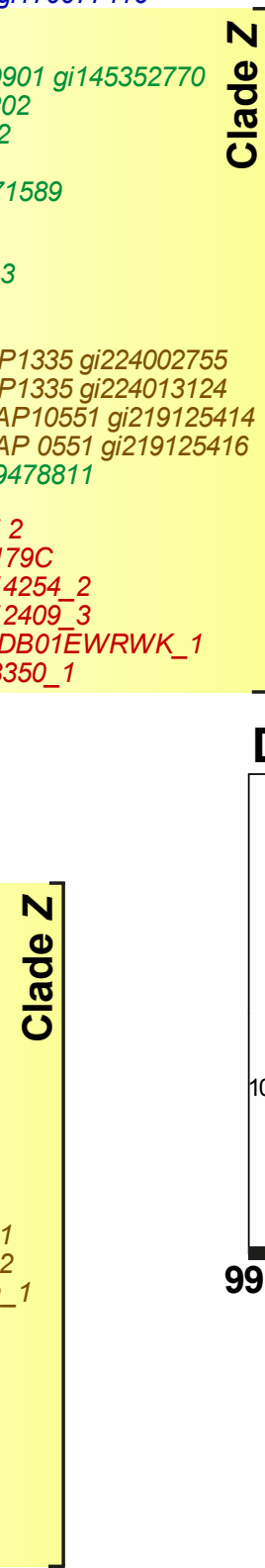

B

Proteobacteria-marine g-proteobacterium

100-Proteobacteria-Marinobacter algicola



1943 -Rhodophyta-Porphyra haitanensis

Rhodophyta-Cyanidioschyzon merolae

99 Viridiplantae-Ostreococcus tauri 1

39100 _ Viridiplantae-Ostreococcus lucimarinus_1

99LViridiplantae-Ostreococcus lucimarinus_2 - Viridiplantae-Micromonas CCMP1545

- Viridiplantae-Micromonas RCC299_1

$6 8 \longdiv { 2 }$-Viridiplantae-Micromonas RCC299_2

35100 91 [ Viridiplantae-Micromonas RCC299 3

62 _ Viridiplantae-Micromonas RCC299_4

100 -Viridiplantae-Ostreococcus lucimarinus_3

_ Viridiplantae-Ostreococcus tauri_2

6082 - Viridiplantae-Ostreococcus RCC809 2

20 Stramenopiles-Thalassiosira pseudonana_1

74 _ Stramenopiles-Phaeodactylum tricornutum_ 1

92 Rhodophyta-Porphyridium purpureum 1

100 - Stramenopiles-Phaeodactylum tricomütum_2

36 Cyanobacteria-Synechococcus sp_2

3639 - Proteobacteria-marine g-proteobacterium

LProteobacteria-Marinobacter sp

29 Stramenopiles-Phaeodactylum tricornutum

100 Viridiplantae-Chlamydomonas reinhardtii

$65-$ Viridiplantae-Volvox carteri

63 Rhodophyta-Porphyridium purpureum_2

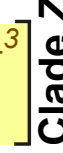

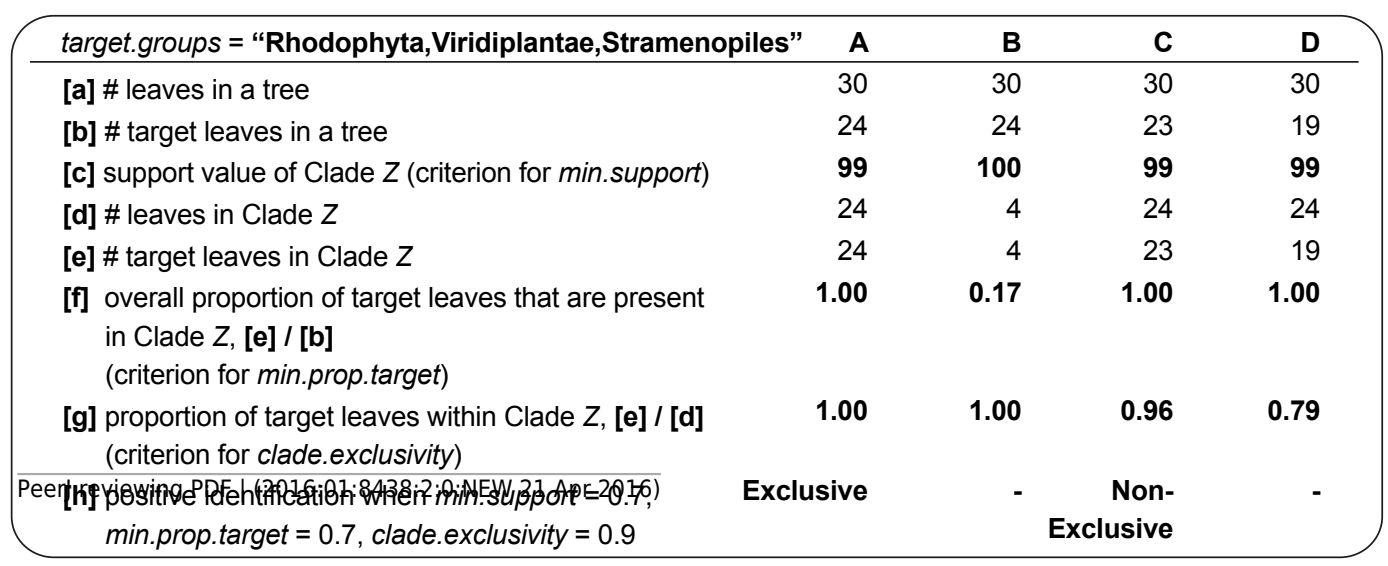

$33\left[\begin{array}{c}100 \\ \text { - Proteobacteria-Marinobacter sp } \\ \text { Proteobacteria-Marinobacter algicola }\end{array}\right.$

19 43-Proteobacteria-Geobacter loveyi

Cyanobacteria-Synechococcus $s p$

99 - Viridiplantae-Ostreococcus tauri 1

- Viridiplantae-Ostreococcus RCC809 1

100 _ Viridiplantae-Ostreococcus lucimariñus_1

Haptophyceae-Emiliania huxleyi_1
Hocidiplantae-Ostreococcus lucimarin

68 Haptophyceae-Emiliania huxleyi_2

100100 91- Haptophyceae-Pavlova Iutheri 1

62 Haptophyceae-Pavlova lutheri_2

100 Viridiplantae-Ostreococcus lucimarinus_3

68 - Viridiplantae-Ostreococcus tauri 2

68 82 - Viridiplantae-Ostreococcus RCC809 2

-Stramenopiles-Thalassiosira pseudoñana_1

99

100 Stramenopiles-Thalassiosira pseudonana_2

74 _- Stramenopiles-Phaeodactylum tricornutum_1

92 L Stramenopiles-Phaeodactylum tricornutum_2

100 -Viridiplantae-Chlamydomonas reinhardtii

95 Viridiplantae-Volvox carteri

$9539-$ Rhodophyta-Porphyra haitanensis

Rhodophyta-Porphyra haitanensis
Rhodophyta-Cyanidioschyzon merolae

99 Rhodophyta-Porphyridium purpureum_1

100 Rhodophyta-Porphyridium purpureum_2

65_-Rhodophyta-Porphyridium purpureum_3

63 -Rhodophyta-Porphyridium purpureum_4

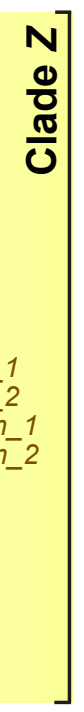

target.groups = "Rhodophyta,Viridiplantae,Stramenopiles"

[a] \# leaves in a tree

A

B 
Figure 2 (on next page)

Overview of the sorting algorithm in PhySortR. 
1 Input: Phylogenetic trees and user-defined sorting parameters

2 Output: Sorted phylogenetic trees

3 for each tree in target directory do
$4 \quad$ read tree
5
if tree is in extended Newick format then
$7 \quad$ end if
$8 \quad$ import tree using ape package
if all taxa in tree are targets then
the tree is "All Exclusive"

\section{end if}

convert into traditional Newick using convert.eNewick

12 for each rooted and unrooted node in tree do

\section{if all target taxa in the node then} the tree is "Exclusive"

if node satisfies min.support, min.prop.target and node has $\geq 1$ taxon from each member of target.groups then

19

\section{end if}

20 end for

\section{1 end for}

else if node satisfies clade.exclusivity then

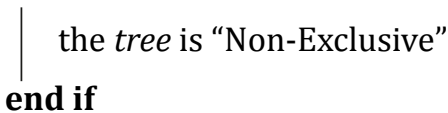

\section{end if}


Figure 3 (on next page)

A phylogenetic tree represented in standard and extended Newick formats. 


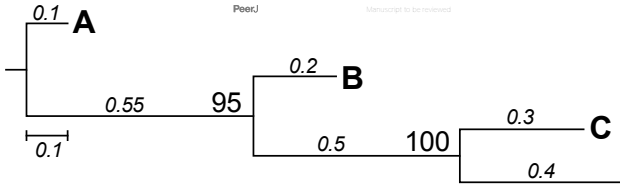

Newick:

(A:0.1, (B:0.2, (C:0.3, D:0.4) 100:0.5) 95:0.55) ;

Extended Newick (eNewick):

$(\mathrm{A}: 0.1,(\mathrm{~B}: 0.2,(\mathrm{C}: 0.3, \mathrm{D}: 0.4) 0.5[100]) 0.55[95])$; 
Figure 4 (on next page)

Benchmarking results of PhySortR using simulated data.

The mean runtime of PhySortR is shown for analysis across datasets (A) with different numbers of trees, $\mathrm{N}$, and $(B)$ with different numbers of leaves per tree, $X$. Values of runtime (in second) are mean across 100 replicates, error bars indicate the standard deviation of the mean. 
Figure 5 (on next page)

Trees sorted using PhySortR and PhyloSort.

The Venn diagram depicting number of trees identified using PhySortR, PhyloSort-root and PhyloSort-no-root is shown in (A). An example of tree output for trees that are identified in (B) all cases, (C) only PhyloSort-root, and (D) all but PhyloSort-no-root are shown. 


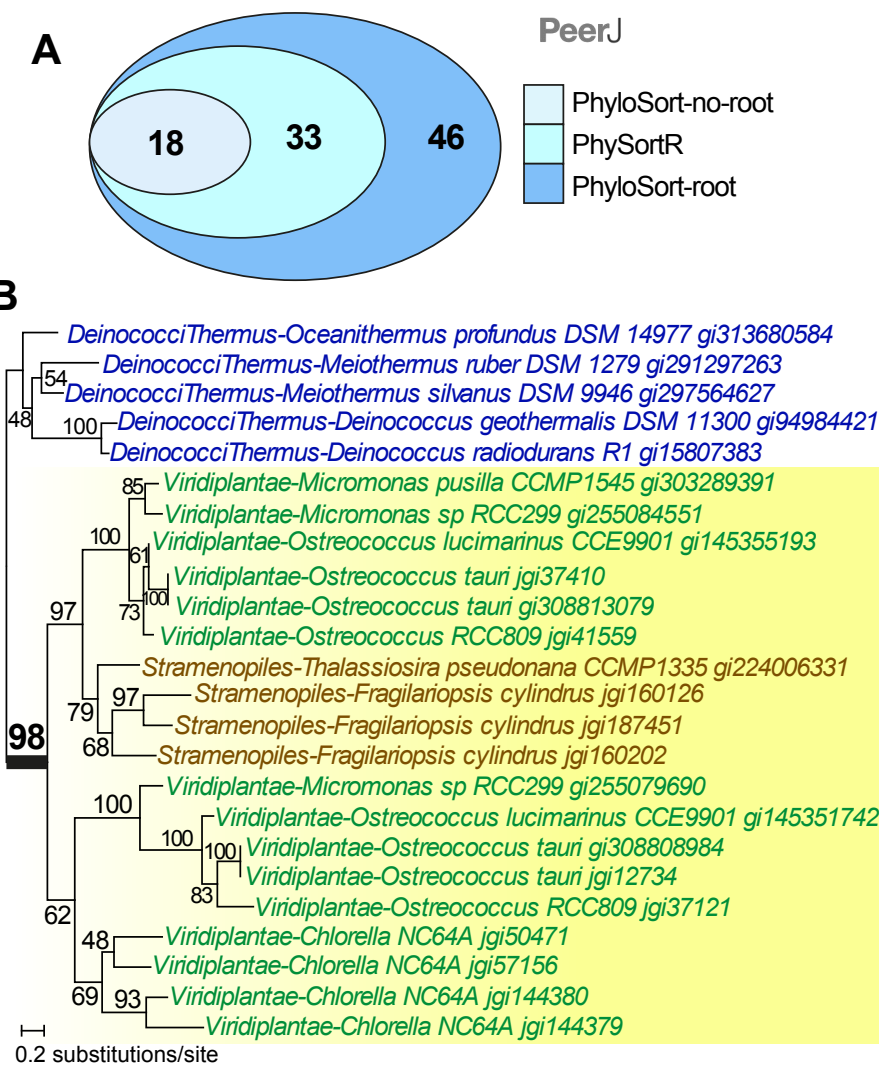

0.2 substitutions/site

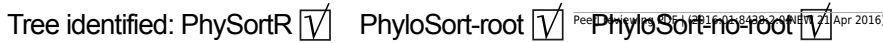

C BacteroidetesChlorobi-Rhodothermus marinus DSM 4252 gi268317919

44 ManMitrospirae-Candidatus Nitrospira defluvi gi302039359

98 Proteobacteria-Geobacter loveyi SZ gi1 189426542

Proteobacteria-Geobacter sp FRC 32 gi222056675

4623 86-Proteobacteria-Geobacter uraniireducens Rf4 gi148263169

39 Proteobacteria-Syntrophobacter fumaroxidans MPOB gi116749888

Proteobacteria-Desulfurivibrio alkaliphilus AHT2 gi297569034

$100 \mid$ Firmicutes-Lactobacillus salivarius ATCC 11741 gi227890691

100 Firmicutes-Lactobacillus salivarius ACS 116 V Col5a gi301299283

100 Firmicutes-Lactobacillus salivarius UCC118 gi90961604

Firmicutes-Lactobacillus ruminis ATCC 25644 gi227526818

39 Aquificae-Hydrogenivirga sp 1285 R1 1 gi163782277

Firmicutes-Dethiobacter alkaliphilus AHT 1 gi225180936

100 —Viridiplantae-Chlorella NC64A jgi139411

Viridiplantae-Chlorella vilgaris jgi25534

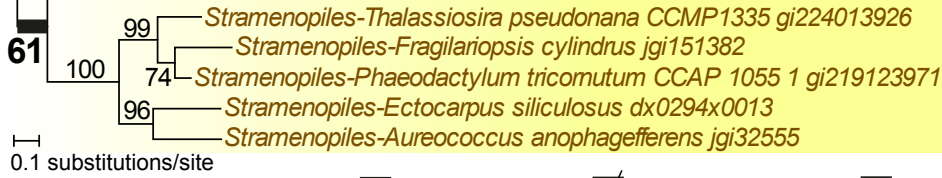

Tree identified: PhySortR $\mathbf{X}$ PhyloSort-root $\square$ PhyloSort-no-root $\mathbf{X}$

D 33 Metazoa-Strongylocentrotus purpuratus gi115772706

$1 9 \longdiv { 3 3 - M e t a z o a - T r i c h o p l a x ~ a d h a e r e n s ~ g i 1 9 6 0 0 9 0 8 7 ~ }$

100 Metazoa-Nematostella vectensis gi156377007

$2519 \quad$ Metazoa-Nematostella vectensis gi156337204

19 Metazoa-Lottia gigantea jgi133911

100 Metazoa-Gallus gallus gi118091252

100 Metazoa-Danio rerio gi82658256

- Metazoa-Salmo salar gi291190226

-Metazoa-Ciona intestinalis gi198433026

$98 \quad 100-$ Vindiplantae-Chlamydomonas reinhardtii gi159478234 - Viridiplantae-Volvox carteri f nagariensis gi302828310

Stramenopiles-Phaeodactylum tricomutum CCAP 10551 gi219122553

100 Stramenopiles-Fragilariopsis cylindrus jgi234967

0.1 substitutions/site

Tree identified: PhySortR $\downarrow$ PhyloSort-root $\downarrow$ PhyloSort-no-root $\mathbf{x}$ 


\section{Table 1 (on next page)}

List of arguments within sortTrees function in PhySortR. 
1 Table 1: List of arguments within sortTrees function in PhySortR.

\begin{tabular}{|c|c|}
\hline Argument & Description \\
\hline target.groups & $\begin{array}{l}\text { A set of one or more terms that represent the target leaves. Multiple terms are to be } \\
\text { separated by a comma and enclosed in quotation marks i.e. } \\
\text { "Rhodophyta,Viridiplantae". This process is case-sensitive; it uses partial string } \\
\text { matching, so the terms used must be unique i.e. "plantae" and "Viridiplantae" are } \\
\text { not appropriate as the first is a subset of the second. }\end{array}$ \\
\hline min.support & $\begin{array}{l}\text { The minimum support (between } 0-1 \text { or } 0-100 \text { inclusive) for any clade identified } \\
\text { during sorting (default } 0 \text { ), dependent on the range of support values noted in the tree } \\
\text { file (e.g. bootstrap support, Bayesian posterior probability, or any similar measure). } \\
\text { A node with no identified support value is treated as having a value of zero }(0) \text {. }\end{array}$ \\
\hline min.prop.target & $\begin{array}{l}\text { The minimum proportion (between } 0.0-1.0 \text { inclusive) of target leaves to be present } \\
\text { in a clade, out of the total target leaves in the tree (default } 0.7 \text { ). At } 0.5, \geq 50 \% \text { of all } \\
\text { target leaves in a tree must be in the clade; at } 1.0 \text {, all target leaves in a tree must be } \\
\text { in the clade. }\end{array}$ \\
\hline in.dir & $\begin{array}{l}\text { The path to the input directory containing all phylogenetic trees to be sorted. If no } \\
\text { value is given, the function defaults to the user's current working directory. }\end{array}$ \\
\hline out.dir & $\begin{array}{l}\text { The output directory to be created within in.dir, for the trees identified during sorting } \\
\text { to be moved or copied to. If } \text { out.dir is omitted, the default directory of Sorted_Trees/ } \\
\text { will be used. If list mode (mode="l") is given, this argument will be ignored, and no } \\
\text { directory will be created. The content of out.dir is dependent on the clades.sorted } \\
\text { parameter. }\end{array}$ \\
\hline mode & $\begin{array}{l}\text { Option to control whether the function will move (" } m \text { "), copy ("c") or list ("l") the } \\
\text { files containing trees identified during sorting. In both move and copy modes the } \\
\text { files will be transferred to subdirectories within out.dir and a list of the sorted trees } \\
\text { will be returned. In the list mode, only the list will be returned. The type of trees } \\
\text { sorted is dependent on the clades.sorted parameter. }\end{array}$ \\
\hline clades.sorted & $\begin{array}{l}\text { Option to control sorting for "Exclusive" (" } E \text { ") or "Non-Exclusive" ("NE") clades. } \\
\text { The default setting is to search for both types of clades, i.e. "E, } N E \text { ". Sorting of } \\
\text { "Exclusive" clades will also generate a sub-group of "All Exclusive" trees (see } \\
\text { Supplementary Text). This argument will affect what is returned by the function and } \\
\text { what subdirectories are created in out.dir. }\end{array}$ \\
\hline extension & The file extension of the input phylogenetic trees (default ".tre"). \\
\hline clade.exclusivity & $\begin{array}{l}\text { The minimum proportion of target leaves allowed in a "Non-Exclusive" clade, } \\
\text { applicable only when sorting } N E \text { clades. The value must be } \geq 0.0 \text { and }<1.0 \text {. At default } \\
(0.9), \geq 90 \% \text { (but not } 100 \%) \text { of the leaves in a } N E \text { clade must be target leaves (i.e. } \\
<10 \% \text { can be "interrupting" non-target leaves). Specification of } 1.0 \text { is not allowed; } \\
1.0 \text { implies that all }(100 \%) \text { leaves in a clade are target leaves (no non-target leaves } \\
\text { allowed), thus the clade would be "Exclusive", not "Non-Exclusive". }\end{array}$ \\
\hline
\end{tabular}

